\title{
Uniform Leader Election Protocols for Radio Networks *
}

\author{
Koji Nakano \\ School of Information Science \\ Japan Advanced Institute of Science Technology \\ Tatsunokuchi, Ishikawa 923-1292, JAPAN \\ nakanodelcom.nitech.ac.jp
}

\author{
Stephan Olariu \\ Department of Computer Science \\ Old Dominion University \\ Norfolk, Virginia 23529, USA \\ olariuecs.odu.edu
}

\begin{abstract}
A radio network is a distributed system with no central arbiter, consisting of $n$ radio transceivers, henceforth referred to as stations. We assume that the stations are identical and cannot be distinguished by serial or manufacturing number. The leader election problem asks to designate one of the station as leader. A leader election protocol is said to be uniform if in each time slot every station transmits with the same probability.

In a seminal paper Willard [9] presented a uniform leader election protocol for single-channel single-hop radio stations terminating in $\log \log n+o(\log \log n)$ expected time slots. It was open whether Willard's protocol featured the same time performance with "high probability". We propose a uniform leader election protocol that terminates, with probability exceeding $1-\frac{1}{f}$ for every $f \geq 1$, in $\log \log n+o(\log \log n)+O(\log f)$ time slots. We also prove that for every $f \in e^{O(n)}$, in order to ensure termination with probability exceeding $1-\frac{1}{f}$, Willard's protocol must take $\log \log n+\Omega(\sqrt{f})$ time slots. Finally, we provide simulation results that show that our leader election outperforms Willard's leader election protocol in practice.
\end{abstract}

\section{Introduction}

A radio network ( $\mathrm{RN}$, for short) is a distributed system with no central arbiter, consisting of $n$ radio transceivers, henceforth referred to as stations. In a single-channel $\mathrm{RN}$ the stations communicate over a unique radio frequency channel known to all the stations. A RN is said to be singlehop when all the stations are within transmission range of each other. In this work we focus on single-channel, single-

\footnotetext{
*Work supported, in part, by the NSF grant CCR-9522093, by ONR grant N00014-97-1-0526, and by a grant from the Telecommunications Advancement Foundation.
}

hop radio networks. Single-hop radio networks are the basic ingredients out of which larger, multi-hop radio networks are built $[1,9]$. As customary, time is assumed slotted and all transmissions are edge-triggered, that is, take place at time slot boundaries [1,3]. In a time slot a station can transmit and/or listen to the channel.

We employ the commonly-accepted assumption that when two or more stations are transmitting on a channel in the same time slot, the corresponding packets collide and are garbled beyond recognition. It is customary to distinguish among radio networks in terms of their collision detection capabilities. In the $\mathrm{RN}$ with collision detection the status of a radio channel in a time slot is, NULL if no station transmitted in the current time slot, SINGLE if exactly one station transmitted in the current time slot, COLLISION if two or more stations transmitted the channel in the current time slot.

The problem that we address in this work is the classical leader election problem which asks to designate one of the station in the network as leader. In other words, after executing the leader election protocol, exactly one station learns that it was elected leader, while the remaining stations learn the identity of the leader.

The leader election problem can be studied in the following three scenarios: Scenario 1 if the number $n$ of stations is known in advance, Scenario 2 if the number $n$ of stations is unknown, but an upper bound $u$ on $n$ is known in advance, and Scenario 3 if neither the number of stations nor an upper bound on this number is known in advance. It is intuitively clear that the task of leader election is the easiest in Scenario 1 and the hardest in Scenario 3, with Scenario 2 being in-between the two.

Randomized leader election protocols designed for single-channel, single-hop radio networks work as follows: in each time slot, the stations transmit on the channel with some probability. As we will discuss shortly, this probability may or may not be the same for individual stations. If the status of the channel is SINGLE, the unique station that has transmitted is declared the leader. If the status is not 
SINGLE, the above is repeated until, eventually, a leader is elected. Suppose that a leader election protocol runs for $t$ time slots and a leader has still not been elected at that time. The history of a station up to time slot $t$ is captured by

the status of the channel: the status of the channel in each of the $t$ time slots, that is, a sequence of $\{$ NULL,COLLISION $\}$ of length $t$.

transmit/not-transmit: The transmission activity of the station in each of the $t$ time slots, that is, a sequence of $\{$ transmit,not-transmit $\}$ of length $t$.

It should be clear that its history contains all the information that a station can obtain in $t$ time slots. From the perspective of how much of the history information is used, we identify three types of leader election protocols for single-channel, single-hop radio networks: em oblivious if, in time slot $i$, $(1 \leq i)$, every station transmits with probability $p_{i}$ and the probability $p_{i}$ is fixed beforehand and does not depend on the history uniform if, in time slot $i,(1 \leq i)$, all the stations transmit with the same probability $p_{i}$, where $p_{i}$ is a function of the history of the status of channel in time slots $1,2, \ldots, i-1$ non-uniform if, in each time slot, every station determines its transmission probability depending on its own history.

Several randomized protocols for single-channel, singlehop networks have been presented in the literature. Metcalfe and Boggs [4] presented an oblivious leader election protocol for Scenario 1 that is guaranteed to terminate in $O(1)$ expected time slots. Their protocol is very simple: every station keeps transmitting on the channel with probability $\frac{1}{n}$. When the status of channel becomes SINGLE, the unique station that has transmitted is declared the leader. Recently, Nakano and Olariu [6] presented two nonuniform leader election protocols for Scenario 3. The first one terminates, with probability $1-\frac{1}{n}$, in $O(\log n)$ time slots ${ }^{1}$. The second one terminates with probability $1-\frac{1}{\log n}$ in $O(\log \log n)$ time slots. The main drawback of these protocols is that the "high probability" expressed by either $1-\frac{1}{n}$ or $1-\frac{1}{\log n}$ becomes meaningless for small values of $n$. For example, the $O(\log \log n)$-time protocol may take a very large number of time slots to terminate. True, this only happens with probability at most $\frac{1}{\log n}$. However, when $n$ is small, this probability is non-negligible.

To address this shortcoming, Nakano and Olariu [7] improved this protocol to terminate, with probability exceeding $1-\frac{1}{f}$, in $\log \log n+2.78 \log f+o(\log \log n+$ $\log f$ ) time slots. Nakano and Olariu [8] also presented an oblivious leader election protocol for Scenario 3 terminating with probability at least $1-\frac{1}{f}$, in $O\left(\min \left((\log n)^{2}+\right.\right.$ $\left.\left.(\log f)^{2}, f^{\frac{3}{5}} \log n\right)\right)$ time slots.

\footnotetext{
${ }^{1}$ In this paper, $\log$ and $\ln$ are used to denote the logarithms to the base 2 and $e$, respectively.
}

In a landmark paper, Willard [9] presented a uniform leader election protocol for the conditions of Scenario 2 terminating in $\log \log u+O(1)$ expected time slots. Willard's protocol involves two stages: the first stage, using binary search, guesses in $\log \log u$ time slots a number $i,(0 \leq i \leq$ $\log u$ ), satisfying $2^{i} \leq n<2^{i+1}$. Once this approximation for $n$ is available, the second stage elects a leader in $O(1)$ expected time slots using the protocol of [4]. Thus, the protocol elects a leader in $\log \log u+O(1)$ expected time slots. Willard [9] went on to improve this protocol to run under the conditions of Scenario 3 in $\log \log n+o(\log \log n)$ expected time slots. The first stage of the improved protocol uses the technique presented in Bentley and Yao [2], which finds an integer $i$ satisfying $2^{i} \leq n<2^{i+1}$, bypassing the need for a known upper-bound $u$ on $n$.

Our first contribution is to propose a uniform leader election protocol terminating, with probability exceeding $1-\frac{1}{f}$, in $\log \log n+o(\log \log n)+O(\log f)$ time slots. Our uniform leader election features the same performance as the non-uniform leader election protocol of [7] even though all the stations transmit with the same probability in each time slot. This protocol is optimal because, as proved by Willard [9], every uniform leader election protocols needs $\log \log n-O(1)$ expected time slots to terminate and, as we will show later, any uniform protocol that elects a leader with probability at least $1-\frac{1}{f}$ needs to run for $\log f$ time slots.

Recall that Willard's uniform leader election protocol [9] runs in $\log \log n+o(\log \log n)$ expected time. Since our uniform leader election protocol runs in $\log \log n+$ $o(\log \log n)$ expected time slots, it features the same performance as Willard's protocol in terms of the expected number of time slots. However, the distribution of the time slots is different in the two protocols. In order to show this fact, we prove that with probability at least $1-\frac{1}{f}$ Willard's protocol has to run for at least $\log \log n+\Omega(\sqrt{f})$ time slots to elect a leader. Thus, Willard's protocol stands a much larger chance than our protocol to run for a long time before electing a leader.

Further, as we are going to show, $\Omega(\sqrt{f})$ is a dominant factor for some applications. Suppose that $n$ stations are partitioned into $\sqrt{n}$ clusters of $\sqrt{n}$ stations each. Let us consider a task involving the following two steps:

Step 1 elect a leader in each cluster;

Step 2 elect a leader among the leaders elected in Step 1.

Note that, all of the $\sqrt{n}$ leaders must be elected in Step 1 before starting Step 2. Using our leader election protocol, Step 1 terminates, with probability at least $1-\frac{1}{f}$, in $\log \log \sqrt{n}+$ $o(\log \log \sqrt{n})+O(\log (f \sqrt{n}))=O(\log n+\log f)$ time slots. 
Step 2 takes $\log \log \sqrt{n}+o(\log \log \sqrt{n})+O(\log f)=$ $\log \log n+o(\log \log n)+O(\log f)$ time slots. Thus, using our leader election protocol, the task can be completed in $O(\log n)$ expected time slots. On the other hand, using Willard's leader election protocol, the expected time slots to complete this task is much larger, even if Willard's leader election protocol runs for $\log \log n+o(\log \log n)+$ $O(\sqrt{f})$. Step 1 takes $\log \log \sqrt{n}+o(\log \log \sqrt{n})+$ $O(\sqrt{f \sqrt{n}})=O\left(n^{\frac{1}{4}}+\sqrt{f}\right)$ time slots. Step 2 takes $\log \log n+o(\log \log n)+O(\sqrt{f})$ time slots. Consequently, using Willard's protocol, the task is completed, with probability $1-\frac{1}{f}$, in $O\left(n^{\frac{1}{4}}+\sqrt{f}\right)$ time slots and thus, in $O\left(n^{\frac{1}{4}}\right)$ expected time slots. Arguably, Willard's protocol is much slower than our protocol to complete this task.

We also provide simulation results that show that our protocol is practically fast and that Willard's uniform leader election protocol is very slow with some probability. More precisely, in 1,000,000 simulations for various values of $n$ up to $100,000,000$, our protocol never required more than 52 time slots, while Willard's protocol needed more than 1,600 time slots in the worst case.

\section{A brief refresher of probability theory}

The main goal of this section is to review elementary probability theory results that are useful for analyzing the performance of our protocols. For a more detailed discussion of background material we refer the reader to [5].

For a random variable $X, E[X]$ denotes the expected value of $X$. Let $X$ be a random variable denoting the number of successes in $n$ independent Bernoulli trials with parameter $p$. It is well known that $X$ has a binomial distribution and that for every integer $r,(0 \leq r \leq n)$,

$$
\operatorname{Pr}[X=r]=\left(\begin{array}{c}
n \\
r
\end{array}\right) p^{r}(1-p)^{n-r} .
$$

Further, the expected value of $X$ is given by

$$
E[X]=\sum_{r=0}^{n} r \cdot \operatorname{Pr}[X=r]=n p .
$$

To analyze the tail of the binomial distribution, we shall make use of the following estimates, commonly referred to as Chernoff bounds [5]:

$$
\begin{aligned}
& \operatorname{Pr}[X>(1+\delta) E[X]]<\left(\frac{e^{\delta}}{(1+\delta)^{(1+\delta)}}\right)^{E[X]}(0 \leq(\boldsymbol{\delta}) \\
& \operatorname{Pr}[X>(1+\epsilon) E[X]]<e^{-\frac{\epsilon^{2}}{3} E[X]} \quad(0 \leq \epsilon \leq 1) \\
& \operatorname{Pr}[X<(1-\epsilon) E[X]]<e^{-\frac{\epsilon^{2}}{2} E[X]}(0 \leq \epsilon \leq 1) .
\end{aligned}
$$

Let $X$ be a random variable assuming only nonnegative values. The following inequality, known as the Markov inequality, will be also used

$$
\operatorname{Pr}[X \geq c \cdot E[X]] \leq \frac{1}{c} \quad \text { for all } c \geq 1 .
$$

To evaluate the expected value of a random variable, we state the following lemma.

Lemma 2.1 Let $X$ be a random variable taking a value smaller than or equal to $T(F)$ with probability at least $F$, $(0 \leq F \leq 1)$, where $T$ is a non-decreasing function. Then, $E[X] \leq \int_{0}^{1} T(F) d F$.

\section{Uniform leader election protocols}

The main purpose of this section is to develop a uniform leader election protocol that terminates, with probability exceeding $1-\frac{1}{f}$, in $\log \log n+o(\log \log n)+O(\log f)$ time slots, where $f \geq 1$ is an arbitrary parameter. We begin by presenting a very simple protocol that is the workhorse of all subsequent leader election protocols.

\section{Protocol Broadcast $(p)$ \\ every station transmits with probability $\frac{1}{2^{p}}$; \\ if the status of the channel is SINGLE then \\ the unique station that has transmitted becomes the \\ leader and all stations exit the (main) protocol}

In Subsection 3.1 we begin by exhibiting a first uniform leader election protocol terminating, with probability exceeding $1-\frac{1}{f}$, in $2 \log \log n+O(\log f)+o(\log \log n)$ time slots. In Subsection 3.2 we show how this protocol can be modified to run in $\log \log n+O(\log f)+o(\log \log n)$ time slots.

\subsection{A uniform leader election protocol running in $2 \log \log n$ time slots}

In outline, our leader election protocol proceeds in three phases.

In Phase 1 the calls Broadcast $\left(2^{0}\right)$, Broadcast $\left(2^{1}\right)$, Broadcast $\left(2^{2}\right), \ldots$, Broadcast $\left(2^{t}\right)$ are performed until, for the first time, the status of the channel is NULL in Broadcast $\left(2^{t}\right)$. At this point Phase 2 begins. Phase 2 executes a variant of binary search on the interval $\left[0,2^{t}\right]$ using the protocol Broadcast as follows:

- First, Broadcast $\left(\frac{2^{t}}{2}\right)$ is executed. If the status of the channel is SINGLE then the unique station that has transmitted becomes the leader.

- If the status of channel is NULL then binary search is performed on the interval $\left[0, \frac{2^{t}}{2}\right]$, that is, Broadcast $\left(\frac{2^{t}}{4}\right)$ is executed. 
- If the status of channel is COLLISION then binary search is performed on the interval $\left[\frac{2^{t}}{2}, 2^{t}\right]$, that is, Broadcast $\left(\frac{3}{4} \cdot 2^{t}\right)$ is executed.

This procedure is repeated until, at some point, binary search cannot further split an interval. Let $u$ be the integer such that the last call of Phase 2 is Broadcast $(u)$. Phase 3 repeats the call Broadcast $(u)$ until, eventually, the status of the channel is SINGLE, at which point a leader has been elected. It is important to note that the value of $u$ is continuously adjusted in Phase 3 as follows: if the status of the channel is NULL, then it is likely that $2^{u}$ is larger than $n$. Thus, $u$ is decreased by one. By the same reasoning, if the status of the channel is COLLISION, $u$ is increased by one.

With this preamble out of the way, we are now in a position to spell out the details of our uniform leader election protocol.

Protocol Uniform-election

Phase 1:

$i \leftarrow-1$;

repeat

$i \leftarrow i+1$

Broadcast $\left(2^{i}\right)$

until the status of the channel is NULL;

Phase 2:

$l \leftarrow 0 ; u \leftarrow 2^{i}$;

while $l+1<u$ do

$m \leftarrow\left\lceil\frac{l+u}{2}\right\rceil ;$

Broadcast $(m)$;

if the status of channel is NULL then

$$
u \leftarrow m
$$

else

$$
l \leftarrow m
$$

endwhile

\section{Phase 3:}

repeat

Broadcast $(u)$;

if the status of channel is NULL then

else

$u \leftarrow \max (u-1,0)$

forever

$u \leftarrow u+1$

We now turn to the task of evaluating the number of time slots it takes the protocol to terminate. In Phase 1, once the status of the channel is NULL the protocol exits the repeat-until loop. Thus, there exist an integer $t$ such that the status of the channel is:

\section{- SINGLE}

or

COLLISION

in the calls Broadcast $\left(2^{0}\right)$, Broadcast $\left(2^{1}\right)$, Broadcast $\left(2^{2}\right), \ldots$, Broadcast $\left(2^{t-1}\right)$, and
- NULL in Broadcast $\left(2^{t}\right)$.

Let $f \geq 1$ be arbitrary and write

$$
s=\lceil\log \log (4 n f)\rceil .
$$

To motivate the choice of $s$ in (5) we show that with probability exceeding $1-\frac{1}{4 f}, s$ provides an upper bound on $t$. Let $X$ be the random variable denoting the number of stations that transmit in Broadcast $\left(2^{s}\right)$. The probability that a particular station is transmitting in the call Broadcast $\left(2^{s}\right)$ is less than $\frac{1}{2^{2^{s}}}$. Thus, the expected value $E[X]$ of $X$ is upper-bounded by

$$
E[X]<\frac{n}{2^{2^{s}}} \leq \frac{n}{4 n f}=\frac{1}{4 f} .
$$

Using the Markov inequality (4) and (6) combined, we can write

$$
\operatorname{Pr}[X \geq 1]<\operatorname{Pr}[X \geq 4 f E[X]] \leq \frac{1}{4 f} .
$$

Equation (7) implies that with probability exceeding $1-$ $\frac{1}{4 f}$, the status of the channel at the end of the call Broadcast $\left(2^{s}\right)$ is NULL confirming that

$$
t \leq s \text { holds with probability exceeding } 1-\frac{1}{4 f} .
$$

Thus, with probability exceeding $1-\frac{1}{4 f}$, Phase 1 terminates in

$t+1 \leq s+1=\lceil\log \log (4 n f)\rceil+1=\log \log n+O(\log \log f)$

time slots. Since Phase 2 terminates in at most $s+1=$ $\log \log n+O(\log \log f)$ time slots, we have proved the following result.

Lemma 3.1 With probability exceeding $1-\frac{1}{4 f}$, Phase 1 and Phase 2 combined take at most $2 \log \log n+$ $O(\log \log f)$ time slots.

Our next goal is to evaluate the value of $u$ at the end of Phase 2. For this purpose, we say that the call Broadcast $(m)$ executed in Phase 2 fails

- if $n \leq \frac{2^{m}}{4(s+1) f}$ and yet the status of the channel is COLLISION, or

- if $n \geq 2^{m} \cdot \ln (4(s+1) f)$ and yet the status of the channel is NULL.

We are interested in evaluating the probability that Broadcast $(m)$ fails. Let $Y$ be the random variable denoting the number of stations transmitting in the call Broadcast $(m)$. First, if $n \leq \frac{2^{m}}{4(s+1) f}$, then $E[Y]=$ 
$\frac{n}{2^{m}} \leq \frac{1}{4(s+1) f}$ holds. By using the Markov inequality (4), we have

$\operatorname{Pr}[Y>1] \leq \operatorname{Pr}[Y>4(s+1) f \cdot E[Y]]<\frac{1}{4(s+1) f}$.

It follows that the status of the channel is COLLISION with probability at most $\frac{1}{4(s+1) f}$.

Next, suppose that $n \geq 2^{m} \cdot \ln (4(s+1) f)$ holds. The status of the channel is NULL with probability at most

$$
\begin{aligned}
\operatorname{Pr}[Y=0] & =\left(1-\frac{1}{2^{m}}\right)^{n} \\
& <e^{-\frac{n}{2^{m}}} \\
& \leq e^{-\ln (4(s+1) f)}=\frac{1}{4(s+1) f}
\end{aligned}
$$

Clearly, in either case, the probability that the call Broadcast $(m)$ fails is at most $\frac{1}{4(s+1) f}$. Importantly, this probability is independent of $m$. Since the protocol Broadcast is called at most $s+1$ times in Phase 2, the probability that none of these calls fails is at least $1-\frac{1}{4 f}$. On the other hand, recall that the probability that Broadcast is called at most $s+1$ times exceeds $1-\frac{1}{4 f}$. Now a simple argument shows that the probability that Phase 2 involves at most $s+1$ calls to Broadcast and that none of these calls fail exceeds $1-\frac{1}{2 f}$. Thus, we have proved the following result.

Lemma 3.2 With probability exceeding $1-\frac{1}{2 f}$, when Phase 2 terminates $u$ satisfies the double inequality $\frac{n}{\ln (4(s+1) f)} \leq$ $2^{u} \leq 4(s+1) f n$.

Finally, we are interested in getting a handle on the number of time slots involved in Phase 3. For this purpose, let $v,(1 \leq v)$, be the integer satisfying the double inequality

$$
2^{v-1}<n \leq 2^{v}
$$

A generic call Broadcast $(u)$ performed in Phase 3 is said to fail to decrease if $u \geq v+2$ and yet the status of the channel is COLLISION, succeed to decrease if $u \geq v+3$ and yet the status of the channel is NULL, fail to increase if $u \leq v-2$ and yet the status of the channel is NULL, succeed to increase if $u \leq v-3$ and yet the status of the channel is COLLISION. good, otherwise.

More generally, we say that the call Broadcast $(u)$ fails if it fails either to increase or to decrease; similarly, the call Broadcast $(u)$ is said to succeed if it succeeds either to increase or to decrease. The motivation for this terminology comes from the observation that if Broadcast $(u)$ succeeds then, $u$ is updated so that $u$ approaches $v$.

Assume that the call Broadcast $(u)$ is good. Clearly, if such is the case, the double inequality $v-2 \leq u \leq v+2$ holds at the beginning of the call. The status of the channel in Broadcast $(u)$ is SINGLE with probability at least

$$
\begin{aligned}
& \left(\begin{array}{c}
n \\
1
\end{array}\right) \frac{1}{2^{u}}\left(1-\frac{1}{2^{u}}\right)^{n-1} \\
& >\frac{n}{2^{u}} e^{-\frac{n}{2^{u}}} \\
& >\min \left(\frac{2^{u+2}}{2^{u}} e^{-\frac{2^{u+2}}{2^{u}}}, \frac{2^{u-3}}{2^{u}} e^{-\frac{2^{u-3}}{2^{u}}}\right) \\
& >\min \left(\frac{4}{e^{4}}, \frac{1}{8 e^{\frac{1}{8}}}\right)=\frac{4}{e^{4}}
\end{aligned}
$$

We note that this probability is independent of $u$. Thus, if a good call is executed $\frac{e^{4}}{4} \ln (4 f)$ times, a leader is elected with probability at least

$$
1-\left(1-\frac{4}{e^{4}}\right)^{\frac{e^{4}}{4} \ln (4 f)}>1-e^{-\ln (4 f)}=1-\frac{1}{4 f} .
$$

As we are about to show, good calls occur quite frequently in Phase 3. To this end, we prove an upper bound on the probability that the call Broadcast $(u)$ fails. Let $Z$ denote the number of stations that transmit in Broadcast $(u)$. Clearly, $E[Z]=\frac{n}{2^{u}}$. Thus, if $u \geq v+2$ then the call Broadcast $(u)$ fails to decrease with probability at most

$$
\begin{aligned}
\operatorname{Pr}[Z>1] & =\operatorname{Pr}\left[Z>\frac{2^{u}}{n} E[Z]\right] \\
& <\operatorname{Pr}\left[Z>\frac{2^{u}}{2^{v}} E[Z]\right] \quad \text { (from } n \leq 2^{v} \text { ) } \\
& <\operatorname{Pr}[Z>4 E[Z]] \quad \text { (from } u \geq v+2) \\
& <\frac{1}{4} \quad \text { (by Markov's inequality (4)) }
\end{aligned}
$$

On the other hand, if $u \leq v-2$ then the probability that Broadcast $(u)$ fails to increase is at most

$$
\begin{aligned}
\operatorname{Pr}[Z=0] & =\left(1-\frac{1}{2^{u}}\right)^{n} \\
& <e^{-\frac{n}{2^{u}}} \\
& <e^{-\frac{2^{v-1} 2^{u}}{4} \quad\left(\text { from } 2^{v-1}<n\right)} \\
& <e^{-2} \quad(\text { from } u \leq v-2) \\
& <\frac{1}{4} .
\end{aligned}
$$

Therefore, the call Broadcast $(u)$ fails with probability at most $\frac{1}{4}$.

Suppose that Broadcast $(u)$ is executed $\frac{8}{3} e^{4}(\ln (4 f)+\log \log \log n)$ times in Phase 3 and let $N_{s}, N_{f}$, and $N_{g}$ be, respectively, the number of times Broadcast $(u)$ succeeds, fails, and is good among these $\frac{8}{3} e^{4}(\ln (4 f)+\log \log \log n)$ calls. Clearly,

$$
N_{s}+N_{f}+N_{g}=4 e^{4}(\ln f+\log \log \log n) .
$$


If at the end of Phase $2 u$ satisfies the double inequality of Lemma 3.2, we have

$$
\begin{aligned}
u & \geq \log \left(\frac{n}{4(s+1) f}\right) \\
& >\log n-\log (s+1)-\log \log f-2 \\
& >v-\log \log f-\log \log \log n-\log \log \log f-4
\end{aligned}
$$

and, similarly,

$$
\begin{aligned}
u \leq & \log (n(\ln (4(s+1) f))) \\
< & \log n+\log \ln (s+1)+\log \ln f+2 \\
< & v+\log \log f+\log \log \log \log n \\
& +\log \log \log \log f+3 .
\end{aligned}
$$

Thus, we have,

$$
|u-v|<2 \log \log f+\log \log \log n+4 .
$$

We note that if $|u-v| \leq 2$ holds at the end of Phase 2, then $N_{s} \leq N_{f}$. By the same reasoning, it is easy to see that if (11) holds at the end of Phase 2, we have

$$
N_{s}<N_{f}+2 \log \log f+\log \log \log n+2 .
$$

Since a particular call Broadcast $(u)$ fails with probability at most $\frac{1}{4}$, we have

$$
E\left[N_{f}\right] \leq \frac{2 e^{4}}{3}(\ln (4 f)+\log \log \log n) .
$$

Thus, the probability that there are more than $e^{4}(\ln (4 f)+$ $\log \log \log n$ ) calls fails is at most

$$
\begin{aligned}
\operatorname{Pr} & {\left[N_{f}>e^{4}(\ln (4 f)+\log \log \log n)\right] } \\
& <\operatorname{Pr}\left[N_{f}>\left(1+\frac{1}{2}\right) E\left[N_{f}\right]\right]<e^{-\frac{1}{2^{2} \cdot 3} E\left[N_{f}\right]} \\
& <e^{-\frac{e^{4}}{24}(\ln (4 f)+\log \log \log n)}<\frac{1}{4 f}
\end{aligned}
$$

Suppose that $N_{f} \leq e^{4}(\log (4 f)+\log \log \log n)$ is satisfied. Then, we have

$$
\begin{aligned}
N_{g}= & \frac{8}{3} e^{4}(\ln (4 f)+\log \log \log n)-\left(N_{s}+N_{f}\right) \\
\geq & \frac{8}{3} e^{4}(\ln (4 f)+\log \log \log n)-2 N_{f} \\
& -(2 \log \log f+\log \log \log n+2) \\
> & \frac{e^{4}}{2} \ln (4 f) .
\end{aligned}
$$

Therefore, with probability at least $1-\frac{1}{4 f}$, among $\frac{e^{4}}{2}(\log (4 f)+\log \log \log n)$ calls Broadcast $(u)$ there are at least $\frac{2}{3} e^{4} \ln (4 f)$ good ones. It follows that if at the end of Phase $2 u$ satisfies the double in equality in Lemma 3.2, then with probability $1-\frac{1}{2 f}$, Phase 3 terminates in at most $\frac{e^{4}}{2}(\log f+\log \log \log n)$ time slots. To summarize, we have proved the following result.
Lemma 3.3 Protocol Uniform-election terminates, with probability at least $1-\frac{1}{f}$, in at most $2 \log \log n+$ $o(\log \log n)+O(\log f)$ time slots.

\subsection{Uniform leader electing protocol running in $\log \log n$ time slots}

The main goal of this subsection is to outline the changes that will make protocol Uniform-election terminate, with probability exceeding $1-\frac{1}{f}$, in $\log \log n+$ $o(\log \log n)+O(\log f)$ time slots.

In

Phase 1 the calls Broadcast $\left(2^{0^{2}}\right)$, Broadcast $\left(2^{1^{2}}\right)$, Broadcast $\left(2^{2^{2}}\right), \ldots$, Broadcast $\left(2^{t^{2}}\right)$ are performed until, for the first time, the status of the channel is NULL in Broadcast $\left(2^{t^{2}}\right)$. Phase 2 performs binary search on $\left[0,2^{t^{2}}\right]$ using Broadcast as discussed in Subsection 3.1. The reader should be in a position to confirm that $t \leq\lceil\sqrt{\log \log (4 n f)}\rceil$ is satisfied with probability at least $1-\frac{1}{f}$, for any $f \geq 1$. Thus, Phase 1 terminates in $\lceil\sqrt{\log \log (4 n f)}\rceil+1$ time slots, while Phase 2 terminates in $(\lceil\sqrt{\log \log (4 n f)}\rceil+1)^{2}$ time slots. Therefore, with probability at least $1-\frac{1}{f}$, Phase 1 and 2 combined terminate in $\log \log n+o(\log \log n)+O(\log \log f)$ time slots. Thus, we have the following result.

Theorem 3.4 There exists a uniform leader election protocol that terminates, with probability at least $1-\frac{1}{f}$, in $\log \log n+o(\log \log n)+O(\log f)$ time slots, for every $f \geq 1$.

It is worth noting that by Lemma 2.1 and Theorem 3.4 combined, our uniform leader election protocol terminates in $\log \log n+o(\log \log n)$ expected time slots.

\section{Willard's uniform leader election protocol}

The main goal of this section is to take a very close look at the performance of Willard's uniform leader election protocol [9]. As it turns out, our uniform leader election protocol presented in Section 3 is very similar to the one in [9]. Both our protocol and Willard's consists of three phases. Phases 1 and 2 are the same for the two protocols. In Willard's protocol, once $u$ is determined at the end of Phase 2, Broadcast $(u)$ is repeated until a leader is elected. Recall that Phase 3 of our protocol keeps adjusting the value of $u$ in each time slot, depending on the status of the channel. On the other hand, Phase 3 of Willard's protocol does not changes the value of $u$.

Quite surprisingly, both our and Willard's protocol feature the exact same expected-time performance, terminating in $\log \log n+o(\log \log n)$ expected time slots. However, as we are going to show, in order to elect a leader with 
probability at least $1-\frac{1}{f}$ for every $f$ such that $f \leq e^{\frac{n+1}{2}}$, Willard's protocol must run for at least $\log \log n+\Omega(\sqrt{f})$ time slots.

Let us consider Willard's version of Uniformelection. In other words, Phase 3 repeats the call Broadcast $(u)$ without changing the value of $u$. Suppose that $n=2^{2^{w}}$ for some integer $w$. Recall that Phase 1 involves the calls Broadcast $\left(2^{0^{2}}\right)$, Broadcast $\left(2^{1^{2}}\right)$, Broadcast $\left(2^{2^{2}}\right), \ldots$, until the status of channel is NULL for the first time.

We assume, without loss of generality, that Phase 1 terminates as a result of the fact that the status of the channel is NULL in the call Broadcast $\left(2^{2^{w}}\right)$ and prove that Phase 3 takes $\Omega(\sqrt{f})$ time slots for this case. The proof of the case where Phase 1 terminates earlier or later is similar and therefore omitted.

After Phase 2, if $u=2^{w}$, then each of the calls in Phase 3 elects a leader with probability at least

$$
\left(\begin{array}{c}
n \\
1
\end{array}\right)\left(1-\frac{1}{2^{m}}\right)^{n-1} \frac{1}{2^{m}}>e^{-\frac{n}{2^{m}}} \frac{n}{2^{m}}>\frac{1}{2 \sqrt{e}} .
$$

It follows that Phase 2 elects a leader in $\frac{1}{\log \left(1-\frac{1}{2 \sqrt{e}}\right)} \log f \approx$ $1.92 \log f$ time slots with probability at least $1-\frac{1}{f}$. However, as we are going to show, Phase 2 does not guarantee that $u=2^{w}$ with high probability.

In order to satisfy $u=2^{w}$ at the end of Phase 2, the status of channel in every call in Phase 2 must be COLLISION. In other words, Broadcast $\left(2^{w}-\frac{2^{w}}{2}\right)$, Broadcast $\left(2^{w}-\right.$ $\left.\frac{2^{w}}{4}\right)$, Broadcast $\left(2^{w}-\frac{2^{w}}{8}\right), \ldots$, Broadcast $\left(2^{w}-1\right)$ should be executed. Let $i$ be any integer satisfying $0 \leq i \leq$ $w$. The status of the channel in the call Broadcast $\left(2^{w}-\right.$ $2^{i}$ ) is NULL with probability at least

$$
\left(1-\frac{1}{2^{2^{w}-2^{i}}}\right)^{n}>\frac{1}{e}\left(1-\frac{2^{2^{i}}}{n}\right)^{n-1}>e^{-2^{2^{i}}-1} .
$$

Once Broadcast $\left(2^{w}-2^{i}\right)$ is NULL then $u$ does not exceed $2^{w}-2^{i}$ at the end of Phase 2. In other words, with probability at least $e^{-2^{2^{i}}-1}$, the inequality $u \leq 2^{w}-2^{i}$ holds after the first phase. Writing $e^{-2^{2^{i}}-1}=\frac{1}{f}$, we have $2^{i}=\log (\ln f-1)$, and $2^{u} \leq 2^{2^{w}-2^{i}}=\frac{n}{\ln f-1}$. Note that $f=e^{2^{2^{i}}+1}$ and $0 \leq i \leq w$, and thus $e^{3} \leq f \leq e^{n+1}$. If the status of the channel in the call Broadcast $\left(2^{w}-\right.$ $\log (\ln f-1))$ is NULL, then a call to Broadcast $(u)$ in Phase 3 elects a leader with probability at most

$$
\begin{aligned}
& \left(\begin{array}{c}
n \\
1
\end{array}\right)\left(1-\frac{1}{2^{u}}\right)^{n-1} \frac{1}{2^{u}} \\
& \leq\left(1-\frac{\ln f-1}{n}\right)^{n-1}(\ln f-1)
\end{aligned}
$$

$$
\begin{aligned}
& <2\left(1-\frac{\ln f-1}{n}\right)^{n}(\ln f-1) \\
& <2(\ln f-1) e^{-(\ln f-1)}<2 e \frac{\ln f-1}{f}
\end{aligned}
$$

Thus, $t$ calls fails to elect a leader with probability at least

$$
\begin{aligned}
& \left(1-2 e \frac{\ln f-1}{f}\right)^{t} \\
& >\left(1-2 e \frac{\ln f-1}{f}\right)\left(1-2 e \frac{\ln f-1}{f}\right)^{t-1} \\
& >\left(1-\frac{4}{e^{2}}\right) e^{-2 e t \frac{\ln f-1}{f}}>e^{-2 e t \frac{\ln f-1}{f}-1}
\end{aligned}
$$

Let $\frac{1}{f}=e^{-2 e t \frac{\ln f-1}{f}-1}$. Then, $t$ must be at least $\Omega(f)$. Hence, with probability at least $\frac{1}{f}$, Phase 3 runs in at least $\Omega(f)$ time slots. Recall that in Phase 2 the status of the channel in the call Broadcast $\left(2^{w}-\log (\ln f-1)\right)$ is NULL with probability $\frac{1}{f}$. Further, if such is the case, Phase 3 runs for at least $f$ time slots with probability at least $1-\frac{1}{f}$. Consequently, Willard's protocol runs in at least $\Omega(f)$ time slots with probability at least $1-\frac{1}{f^{2}}$ for every $e^{3} \leq f^{2} \leq e^{n+1}$. Therefore, we have the following result.

Lemma 4.1 Willard's uniform leader protocol runs in at least $\Omega(\sqrt{f})$ time slots with probability at least $1-\frac{1}{f}$ for every $f \leq e^{\frac{n+1}{2}}$.

\section{Simulation results}

The main goal of this section is to offer yet another perspective of the relative performance of Willard's and our uniform leader election protocols. Both protocols were simulated and the results are captured in Tables 1 and 2 in the form of a histogram. Each protocol was run 1,000,000 times for each of the values

$$
n=2,10,100,1000,10000,100000 \text {, and } 1000000 .
$$

It is easy to see that the two uniform leader election protocols have almost the same performance in terms of the expected number of time slots. However, the simulation results show that Willard's protocol is extremely slow with some probability as we have proved mathematically. In this simulation among the 1,000,000 runs, the largest number of time slots taken by Willard's protocol exceeds 1,600 , while our leader election never takes more than 56 time slots.

\section{References}

[1] R. Bar-Yehuda, O. Goldreich, and A. Itai, Efficient emulation of single-hop radio network with collision 
Table 1. The performance of Willard's uniform leader election protocol

\begin{tabular}{|c|c|c|c|c|c|c|c|}
\hline$n=$ & 2 & 10 & 100 & 1000 & 10000 & 100000 & 1000000 \\
\hline 1 & 500311 & 9671 & 0 & 0 & 0 & 0 & 0 \\
\hline 2 & 218611 & 185948 & 0 & 0 & 0 & 0 & 0 \\
\hline 3 & 117264 & 272504 & 10498 & 0 & 0 & 0 & 0 \\
\hline 4 & 66842 & 159747 & 262230 & 78310 & 0 & 0 & 0 \\
\hline 5 & 40275 & 96389 & 90190 & 13632 & 130218 & 332396 & 8 \\
\hline 6 & 23098 & 93260 & 206693 & 246269 & 0 & 6 & 248 \\
\hline 7 & 13257 & 56369 & 116681 & 57679 & 310615 & 160351 & 3625 \\
\hline 8 & 8051 & 40112 & 96266 & 138792 & 76010 & 31610 & 28829 \\
\hline 9 & 4833 & 26302 & 63966 & 121008 & 128376 & 62030 & 285704 \\
\hline 10 & 2825 & 17789 & 44258 & 99221 & 113483 & 82863 & 178228 \\
\hline 11 & 1692 & 12082 & 30465 & 68730 & 76535 & 100160 & 146388 \\
\hline 12 & 1132 & 7922 & 21013 & 47703 & 51356 & 71373 & 107042 \\
\hline 13 & 669 & 5641 & 14823 & 33794 & 34769 & 48303 & 72967 \\
\hline 14 & 426 & 3980 & 10423 & 23684 & 23081 & 32846 & 50350 \\
\hline 15 & 233 & 2883 & 7464 & 17030 & 15689 & 22603 & 35477 \\
\hline 16 & 155 & 2037 & 5371 & 12164 & 10970 & 15419 & 24585 \\
\hline 17 & 119 & 1429 & 4048 & 8800 & 7651 & 10845 & 17614 \\
\hline 18 & 50 & 1127 & 3033 & 6718 & 5434 & 7671 & 12292 \\
\hline 19 & 61 & 796 & 2178 & 4861 & 3733 & 5349 & 8892 \\
\hline 20 & 32 & 654 & 1739 & 3721 & 2692 & 3974 & 6545 \\
\hline 21 & 15 & 518 & 1410 & 2827 & 1989 & 2866 & 4732 \\
\hline 22 & 16 & 390 & 1049 & 2198 & 1479 & 2064 & 3511 \\
\hline 23 & 8 & 351 & 888 & 1768 & 1091 & 1551 & 2647 \\
\hline 24 & 6 & 267 & 658 & 1471 & 767 & 1136 & 2002 \\
\hline 25 & 2 & 205 & 598 & 1197 & 617 & 875 & 1607 \\
\hline 26 & 5 & 165 & 449 & 936 & 463 & 669 & 1202 \\
\hline 27 & 4 & 140 & 385 & 826 & 396 & 531 & 895 \\
\hline 28 & 0 & 106 & 310 & 699 & 328 & 406 & 734 \\
\hline 29 & 1 & 83 & 273 & 547 & 266 & 308 & 517 \\
\hline 30 & 1 & 79 & 241 & 490 & 212 & 283 & 448 \\
\hline 31 & 1 & 72 & 181 & 487 & 179 & 217 & 339 \\
\hline 32 & 1 & 51 & 154 & 349 & 149 & 177 & 291 \\
\hline 33 & 1 & 52 & 161 & 328 & 134 & 157 & 227 \\
\hline 34 & 0 & 33 & 109 & 301 & 113 & 129 & 193 \\
\hline 35 & 1 & 40 & 102 & 257 & 89 & 98 & 151 \\
\hline 36 & 0 & 32 & 87 & 255 & 81 & 107 & 131 \\
\hline 37 & 0 & 16 & 79 & 219 & 73 & 77 & 118 \\
\hline 38 & 0 & 32 & 70 & 196 & 68 & 68 & 95 \\
\hline 39 & 0 & 21 & 61 & 196 & 51 & 61 & 73 \\
\hline 40 & 0 & 13 & 48 & 153 & 42 & 55 & 76 \\
\hline $41-50$ & 1 & 105 & 331 & 1191 & 319 & 251 & 365 \\
\hline $51-60$ & 1 & 71 & 146 & 495 & 184 & 53 & 143 \\
\hline 61- 70 & 0 & 32 & 104 & 240 & 90 & 24 & 93 \\
\hline 71- 80 & 0 & 49 & 72 & 123 & 66 & 9 & 55 \\
\hline $81-90$ & 0 & 35 & 72 & 45 & 41 & 6 & 41 \\
\hline $91-100$ & 0 & 33 & 67 & 26 & 29 & 0 & 42 \\
\hline $101-200$ & 0 & 236 & 367 & 35 & 71 & 20 & 206 \\
\hline $201-300$ & 0 & 76 & 124 & 11 & 1 & 3 & 100 \\
\hline $301-400$ & 0 & 41 & 41 & 7 & 0 & 0 & 61 \\
\hline $401-500$ & 0 & 6 & 16 & 2 & 0 & 0 & 29 \\
\hline $501-600$ & 0 & 4 & 6 & 1 & 0 & 0 & 29 \\
\hline $601-700$ & 0 & 3 & 0 & 3 & 0 & 0 & 18 \\
\hline $701-800$ & 0 & 0 & 1 & 1 & 0 & 0 & 15 \\
\hline $801-900$ & 0 & 1 & 0 & 1 & 0 & 0 & 5 \\
\hline $901-1000$ & 0 & 0 & 0 & 1 & 0 & 0 & 8 \\
\hline $1001-1100$ & 0 & 0 & 0 & 1 & 0 & 0 & 1 \\
\hline $1101-1200$ & 0 & 0 & 0 & 1 & 0 & 0 & 2 \\
\hline $1201-1300$ & 0 & 0 & 1 & 0 & 0 & 0 & 1 \\
\hline $1401-1500$ & 0 & 0 & 0 & 0 & 0 & 0 & 1 \\
\hline $1501-1600$ & 0 & 0 & 0 & 0 & 0 & 0 & 1 \\
\hline $1601-1700$ & 0 & 0 & 0 & 0 & 0 & 0 & 1 \\
\hline AVE & 2.185 & 4.677 & 7.043 & 8.975 & 8.984 & 8.870 & 11.582 \\
\hline
\end{tabular}

detection on multi-hop radio network with no collision detection, Distributed Computing, 5, (1991), 67-71.

[2] J. Bentley and A. Yao, An almost optimal algorithm for unbounded search, Information Processing Letters, 5, (1976), 82-87.

[3] D. Bertzekas and R. Gallager, Data Networks, Second Edition, Prentice-Hall, 1992.

[4] R. M. Metcalfe and D. R. Boggs, Ethernet: distributed packet switching for local computer networks, Communications of the ACM, 19, (1976), 395-404.

[5] R. Motwani and P. Raghavan, Randomized Algorithms, Cambridge University Press, 1995.
Table 2. The performance of our uniform leader election protocol

\begin{tabular}{|r|rrrrrrr|}
\hline$n=$ & 2 & 10 & 100 & 1000 & 10000 & 100000 & 1000000 \\
\hline 1 & 500306 & 9767 & 0 & 0 & 0 & 0 & 0 \\
2 & 218693 & 185832 & 0 & 0 & 0 & 0 & 0 \\
3 & 77879 & 272252 & 10500 & 0 & 0 & 0 & 0 \\
4 & 94007 & 166660 & 261358 & 78236 & 0 & 0 & 0 \\
5 & 24528 & 99181 & 90097 & 13523 & 130451 & 331851 & 4 \\
6 & 40459 & 94877 & 207357 & 246054 & 0 & 14 & 237 \\
7 & 9803 & 49330 & 116821 & 57902 & 310312 & 160656 & 3784 \\
8 & 16231 & 41589 & 102912 & 142546 & 75895 & 31535 & 28996 \\
9 & 4125 & 23664 & 63662 & 124939 & 128710 & 62445 & 286019 \\
10 & 6643 & 19146 & 44339 & 100638 & 114721 & 77610 & 178482 \\
11 & 1655 & 10970 & 31486 & 72625 & 72921 & 102026 & 145891 \\
12 & 2665 & 9019 & 21803 & 48872 & 52176 & 71009 & 106619 \\
13 & 680 & 5239 & 15208 & 35224 & 34301 & 50991 & 76610 \\
14 & 1103 & 4310 & 10569 & 23811 & 24930 & 33729 & 53468 \\
15 & 273 & 2466 & 7372 & 17211 & 16699 & 23946 & 36242 \\
16 & 460 & 1966 & 4988 & 11552 & 12190 & 16323 & 25502 \\
17 & 97 & 1112 & 3467 & 8325 & 7986 & 11605 & 17540 \\
18 & 196 & 860 & 2454 & 5474 & 5811 & 8026 & 12303 \\
19 & 38 & 521 & 1773 & 3988 & 3870 & 5600 & 8549 \\
20 & 88 & 401 & 1195 & 2787 & 2785 & 3822 & 6153 \\
21 & 13 & 251 & 832 & 1904 & 1809 & 2663 & 4155 \\
22 & 29 & 199 & 538 & 1262 & 1352 & 1815 & 2879 \\
23 & 6 & 121 & 390 & 939 & 951 & 1337 & 1998 \\
24 & 11 & 85 & 286 & 631 & 663 & 918 & 1363 \\
25 & 2 & 50 & 163 & 446 & 426 & 636 & 968 \\
26 & 2 & 43 & 133 & 306 & 322 & 445 & 693 \\
27 & 1 & 36 & 95 & 262 & 222 & 327 & 461 \\
28 & 4 & 21 & 59 & 165 & 129 & 212 & 331 \\
29 & 1 & 10 & 29 & 102 & 116 & 150 & 236 \\
30 & 1 & 7 & 30 & 85 & 70 & 96 & 156 \\
31 & 0 & 5 & 21 & 69 & 51 & 61 & 99 \\
32 & 1 & 6 & 23 & 49 & 33 & 47 & 67 \\
33 & 0 & 2 & 7 & 23 & 28 & 30 & 56 \\
34 & 0 & 0 & 6 & 19 & 27 & 21 & 45 \\
35 & 0 & 0 & 7 & 10 & 11 & 12 & 23 \\
36 & 0 & 2 & 4 & 6 & 12 & 14 & 17 \\
37 & 0 & 0 & 2 & 5 & 4 & 6 & 15 \\
38 & 0 & 0 & 4 & 1 & 4 & 8 & 14 \\
39 & 0 & 0 & 2 & 2 & 3 & 5 & 5 \\
40 & 0 & 0 & 4 & 3 & 3 & 2 & 6 \\
41 & 0 & 0 & 0 & 2 & 1 & 0 & 3 \\
42 & 0 & 0 & 0 & 0 & 0 & 0 & 3 \\
43 & 0 & 0 & 0 & 0 & 1 & 1 & 2 \\
44 & 0 & 0 & 0 & 1 & 0 & 1 & 3 \\
45 & 0 & 0 & 2 & 1 & 2 & 0 & 1 \\
46 & 0 & 0 & 2 & 0 & 0 & 0 & 0 \\
47 & 0 & 0 & 0 & 0 & 1 & 0 & 1 \\
48 & 0 & 0 & 0 & 0 & 0 & 1 & 1 \\
49 & 0 & 0 & 0 & 0 & 0 & 1 & 0 \\
50 & 0 & 0 & 0 & 0 & 0 & 2 & 0 \\
52 & 0 & 0 & 0 & 0 & 1 & 1 & 0 \\
\hline AVE & 2.310 & 4.510 & 6.795 & 8.672 & 8.921 & 8.834 & 11.321 \\
\hline & & & & & & &
\end{tabular}

[6] K. Nakano and S. Olariu, Randomized $O(\log \log n)$ round leader election protocols in radio networks, Proc. of International Symposium on Algorithms and Computation, (LNCS 1533), 209-218, 1998.

[7] K. Nakano and S. Olariu, Randomized leader election protocols for ad-hoc networks, Proc. of Sirocco 7, June 2000, 253-267.

[8] K. Nakano and S. Olariu, Randomized leader election protocols in radio networks with no collision detection, Proc. of International Symposium on Algorithms and Computation, 362-373, 2000.

[9] D. E. Willard, Log-logarithmic selection resolution protocols in a multiple access channel, SIAM Journal on Computing, 15, (1986), 468-477. 